\title{
ORIENTATION SYSTEM OF PV PANELS AFTER SUN
}

\author{
GEORGE EDUARD HOLMAN ${ }^{* 1}$, CIPRIAN AFANASOV ${ }^{1}$ \\ I "Stefan cel Mare” University of Suceava, Strada Universitatii 13, Suceava, 720229 \\ Romania
}

\begin{abstract}
The purpose of the project is create a PV panels orientation system after the sun, with return to home position when dark is coming. The system must be energetically autonomous, and also have the ability to constantly provide power. The orientation of photovoltaic panels is an important factor that we must take into account in the design of energy production systems based on solar radiation. Solar guidance systems increase the efficiency of the power generation system by keeping the panels in a position perpendicular to the light rays. The two-axis orientation method was used. The wiring diagram has been designed so that the guidance system works in both manual and automatic modes. For the system to be energy-independent and, linked to the power supply of the drive motors for the two axis, we used a MPPT charger for the battery used as a energy source. Finding the best solutions for increasing system efficiency and energy independence will be presented in this paper.
\end{abstract}

Keywords: PV panel, positioning, solar tracker, actuators, photoresistors, charge controller, accumulators, storage

\section{INTRODUCTION}

One of the problems of mankind at present is the global energy crisis. A solution to this problem would be to use as many sources of renewable energy as possible. The use of renewable energy sources also solves the problem of pollution and carbon dioxide emissions in the case of the use of energy from solid fuels. Solar energy is the energy transmitted by the sun in the form of heat and light. It is utilized in a number of technologies such as: solar heating, photovoltaic cells, solar thermal energy [1].

The energy collected by the solar panel depends to a large extent on the angle formed between the light beam and the solar panel. Panel yield is maximum when the angle between the light beam and the panel is 90 degrees. For example, if a tracer has an accuracy of $\pm 5^{\circ}$, it gives the panel the ability to capture $99.6 \%$ of direct power plus $100 \%$ of diffuse light. In the last two decades, photovoltaic cells have evolved from the niche market with small-scale applications, becoming a mass of electricity [2].

The first photovoltaic cell was built by Charles Fritts in 1880. In 1931 a German engineer, Dr. Bruno Lange, developed a photovoltaic cell using selenium instead of copper oxide. It has now reached a photovoltaic cell that converts the sun's radiation into electricity at about $44.7 \%$. There is a wide range of solar energy concentrating technologies among the most developed ones: parabolic gutters, linear Fresnel reflector, Stirling vessel and solar energy tower. In all these systems the working fluid is heated by concentrated solar light, and is then used to produce energy or store energy [3].

\footnotetext{
* Corresponding author, email: jaysyang68@gmail.com

(c) 2017 Alma Mater Publishing House
} 
The orientation of the solar panels is an important factor that we must take into account in the design of solar systems. Solar guidance systems increase the efficiency of solar panels, keeping the panels in a position perpendicular to the light rays [4].

Trackers can be graded according to the number and orientation of the axes. These may be, with a single axis, and two axes. In the case of the project, a two-axis tracking device was used. These, unlike those with one axis, have two angles of movement, so two axes of rotation. These trackers allow an optimal energy level for the solar panel as they are capable of tracking the sun both vertically and horizontally [5].

By comparing the performance of fixed systems with those with a tracker on a single axis it is $30 \%$ smaller. In the case of two-axis trackers, the yield is $36 \%$ [6].

The sun changes its position from East to West, influencing the yield of solar energy capture. The maximum yield of photovoltaic panels is in the moment when the sun's rays are perpendicular to the solar panel [7].

\section{EXPERIMENTAL SETUP}

\subsection{Block diagram of the experiment stand}

Figure 1 shows the block diagram of the experimental stand with the following components.

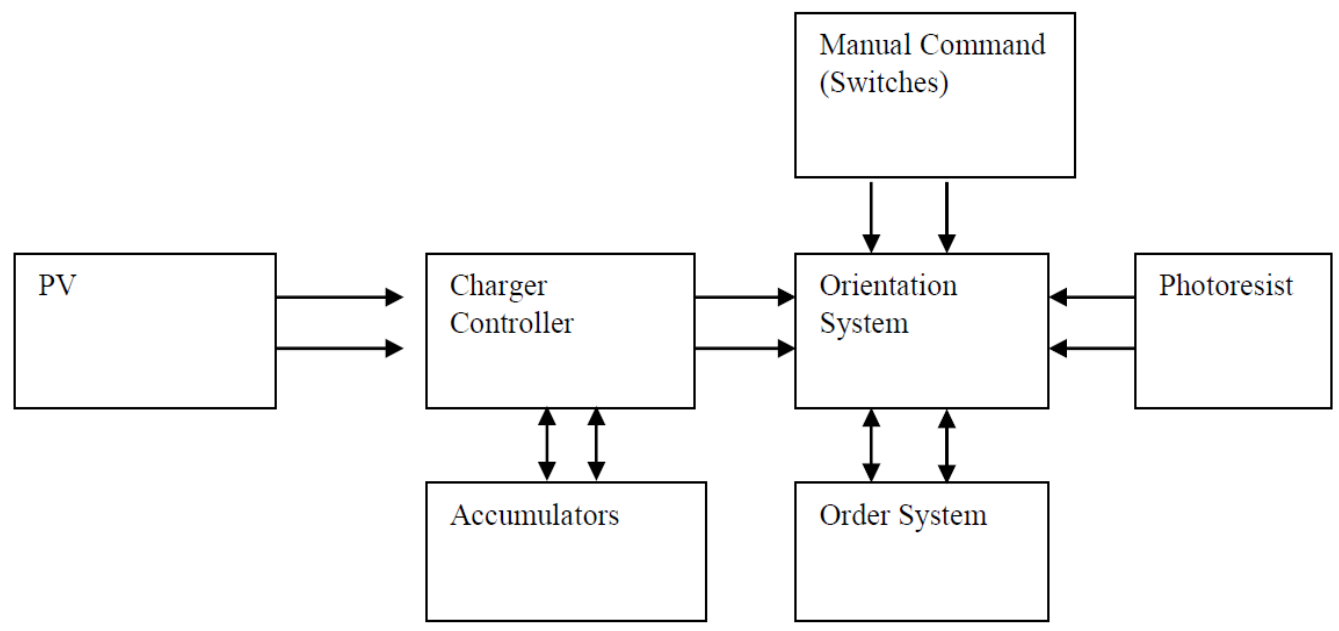

Fig. 1. Components that make up the experimental stand.

As light sensors we use four photoresists, two for each axis. Practically orientation will be achieved with two independent tracker circuits, each track achieving one axis orientation [8].

The solar panel communicates with the charging controller, delivering the maximum energy it can produce. The load controller is linked to what is produced, what is consumed and what it stores. It will be parameterized according to the system you want, and to limit battery download, when to charge the battery, load limits etc. [9]

The orientation system is driven by two actuators, which are controlled by the control through MOSFET transistors, or manual mode [10]

Figure 2 shows the wiring made with the four photoresists two for each axis, and the two additional photoresistors are used to bring the panel into its original position when the darkness. 


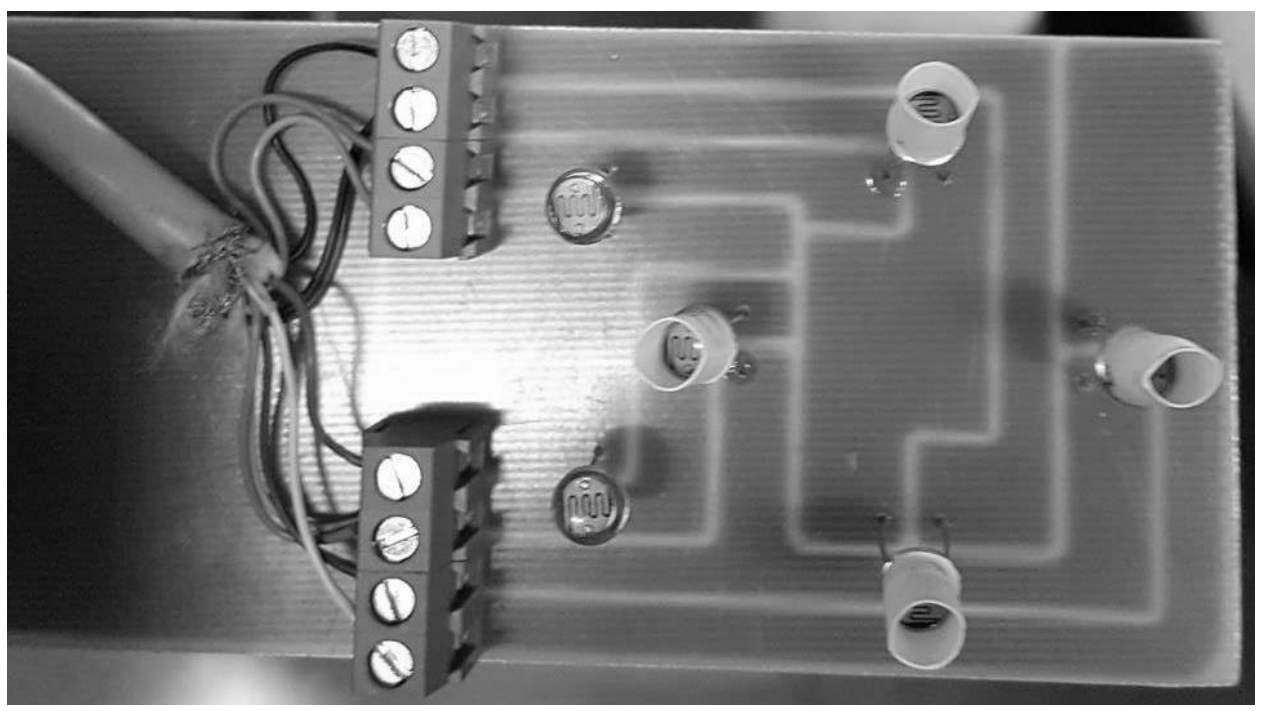

Fig. 2. Photo-resistive wiring.

\subsection{Electrical tracker diagram}

The tracker's electrical scheme is shown in Figure 3.

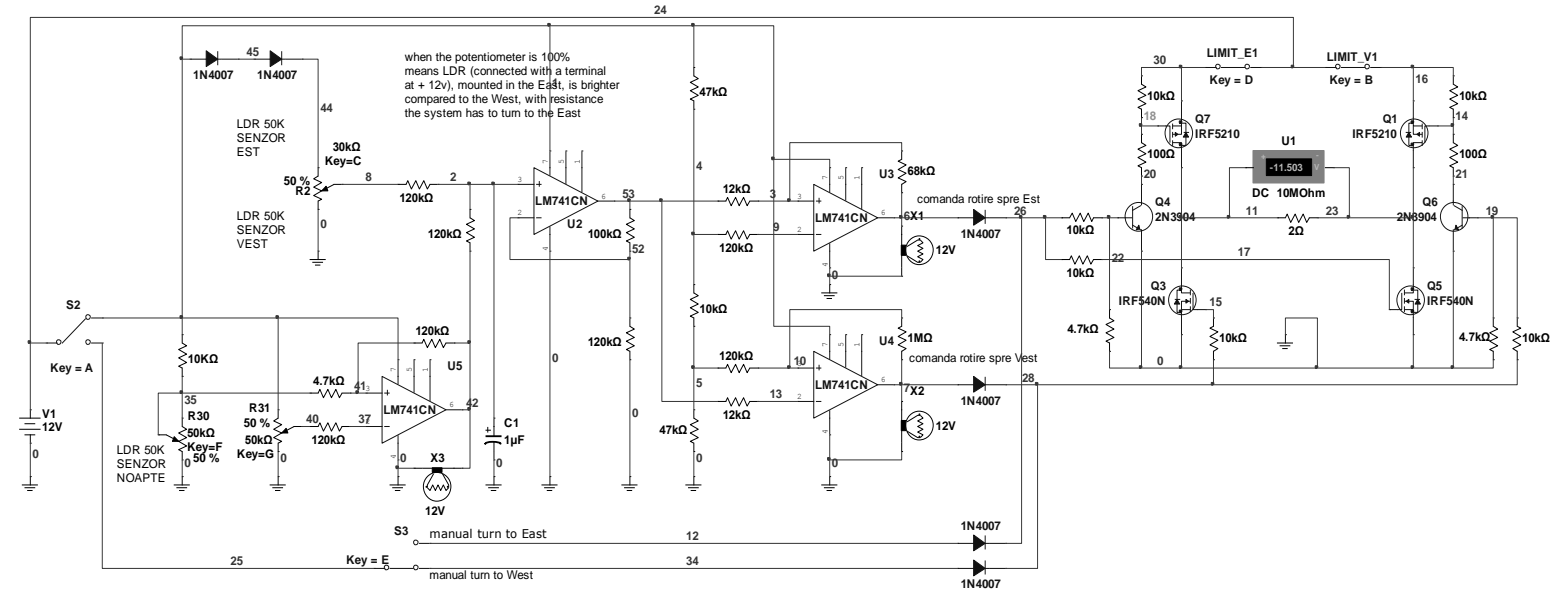

Fig. 3. Electrical tracker diagram.

The wiring diagram has been designed so that the guidance system works in two ways:

- manual mode - the system will be manually oriented, having the possibility of orient it up or down and east west;

- automatic mode - the system will be driven by two actuators, the position of the sun is read by four photoresists two for each axis that need must balanced.

Resistances are grouped two by two. The signals of each pair are compared, making them equal for the system with face the sun. Once the sun moves, one of the photoresistor will shade and one will face the sun. The signals are different, the system will rotate in the proper direction until the resistances are be equal.

The automatic guidance system works as long as the light sensing light sensitivity gives a lesser signal than the signal set by a potentiometer. So with a potentiometer we can select the level of darkness until the targeting system works. When reaching the threshold of darkness, the system will be ordered to go home. The home position is represented by the position of the morning panel when the sunrise the sun.

For motors to work all the time, the guiding system has fixed a hysteresis. This hysteresis allows the system a slight deviation from the sun. 


\subsection{The solar tracker harness}

Figure 4 shows the solar tracker harness made. The tracker system has two identical schemes, one for each axis, the mode of operation being the same.

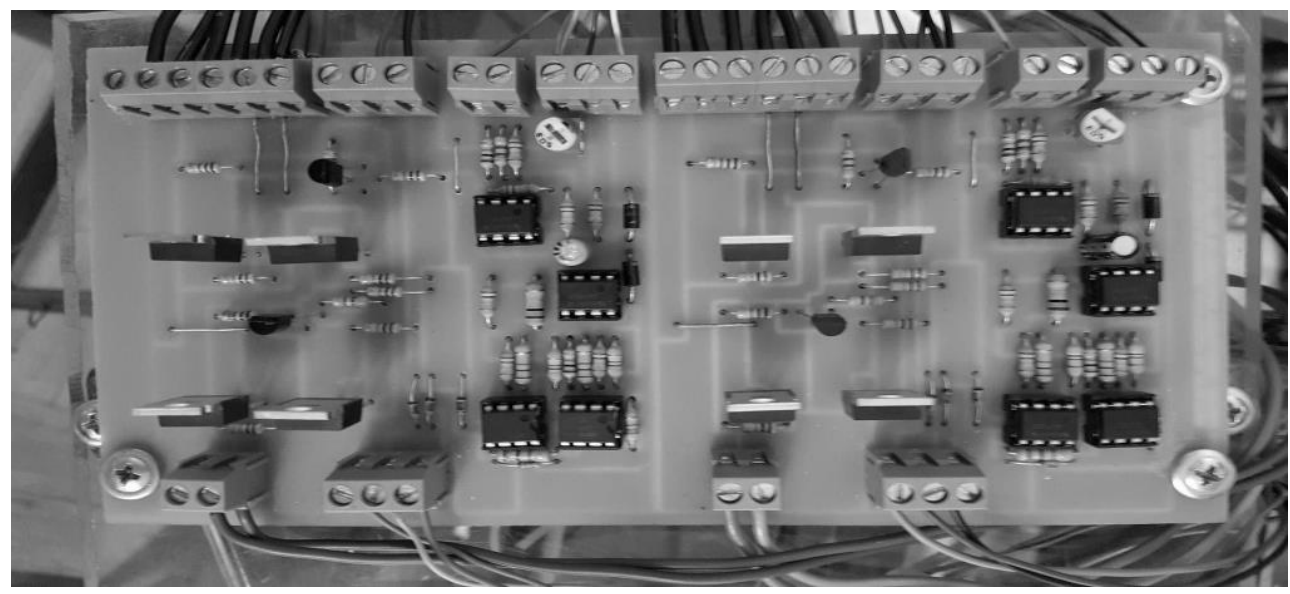

Fig. 4. Tracker wiring.

\subsection{Charge controller}

The Charge Controller is a device that helps us get the most out of our solar system. It is the link between the solar system and the electricity storage system. With the help of we can select when to charge the batteries, at what loading level we want to keep them etc.

The controller has the Maximum Power Point Tracking (MPPT) technology, which has an efficiency of not less than $99.5 \%$. This, together with high-quality components, contributes to the improvement of the system, with a maximum conversion efficiency of $98 \%$. Due to its non-linear characteristics, the solar system has a maximum power point on the curve.

Charge Switch and PWM technology can not capture the maximum power of the system instead, the MPPT controller will lock on the maximum power point and deliver the battery the maximum energy produced by the solar system. With this controller we can delight exactly the types of accumulators used, and with the help of the application it is possible to monitor precisely their loading and unloading cycle.

Figure 5 is the maximum power point curve, the shaded area charging range of traditional solar charge controller PWM Charging Mode, it can obviously diagnose that the MPPT mode can improve the usage of the solar energy resource. According to our test, the MPPT controller can raise $20 \%$ - 30\% efficiency compared to the PWM controller. Value may be fluctuant due to the influence of the ambient circumstance and energy loss.

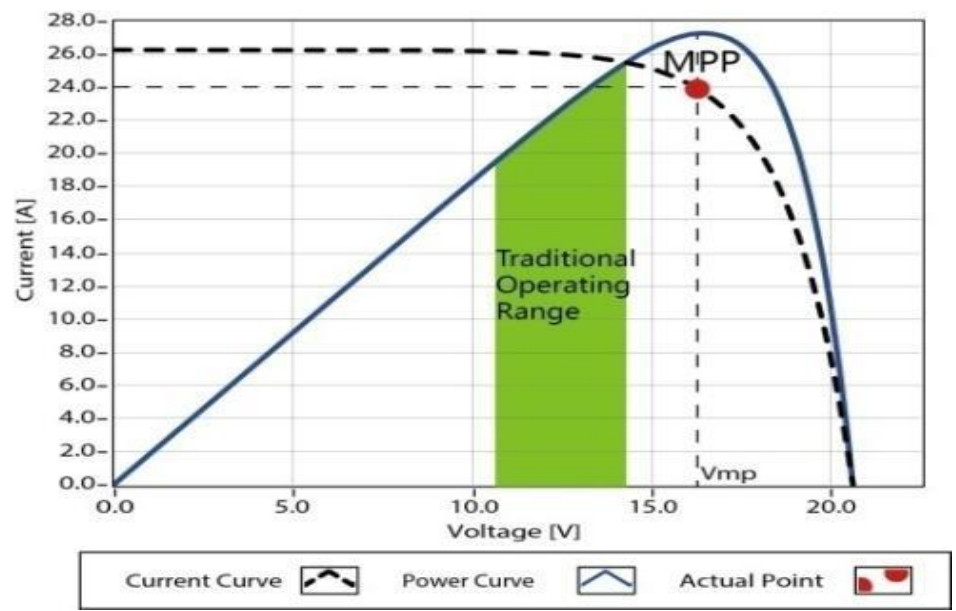

Fig. 5. Maximum power curve. 


\section{RESULTS AND DISCUSSION}

\subsection{System operating graph}

Figure 6 shows the system operating diagram and imposed hysteresis representing the limit of deviation of the system from the position of the sun.

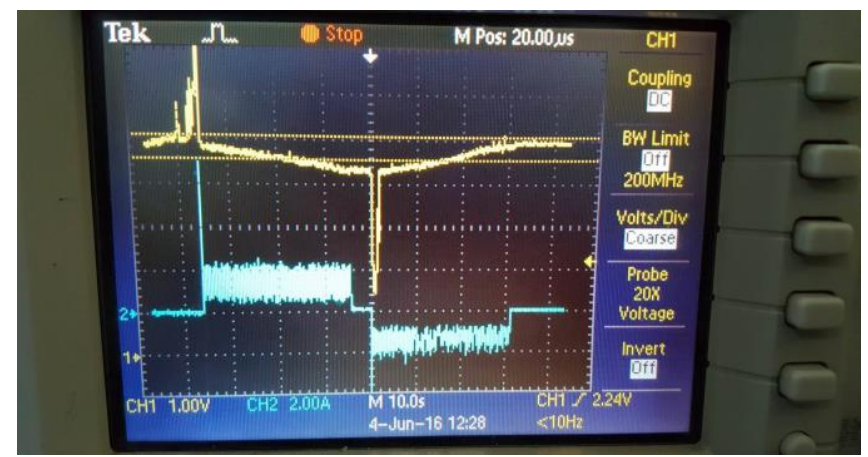

Fig. 6. System operating graph for one of the axes.

On channel 1 we have the command signal from two of the photoresistors and on the second channel we had the current cut by one of the system motors.

It can be seen that when one of the photocells is shaded and the signal rises above the limit of a prescribed threshold, the system starts to return the system to the prescribed range. It is remarkable that the system does not stop at the input of the signal in the prescribed range but it works until reaching the other limit.

The same thing happens if the other cell is shaded. The system will start at reaching the lower limit, and the signal stops will exceed the other limit.

\subsection{The controller behaves at different levels of battery charge}

In the graphs adjacent to Figure 7 and figure 8 we can observe the behavior of the controller when the battery charge level is $70 \%$ and a load is gradually connected.

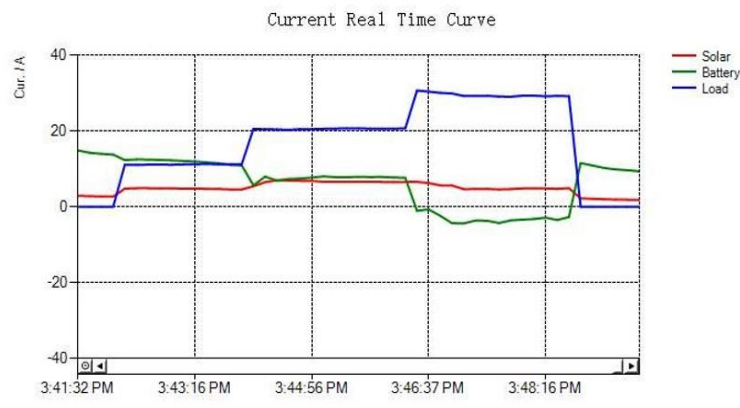

Fig. 7. Current real time curve.

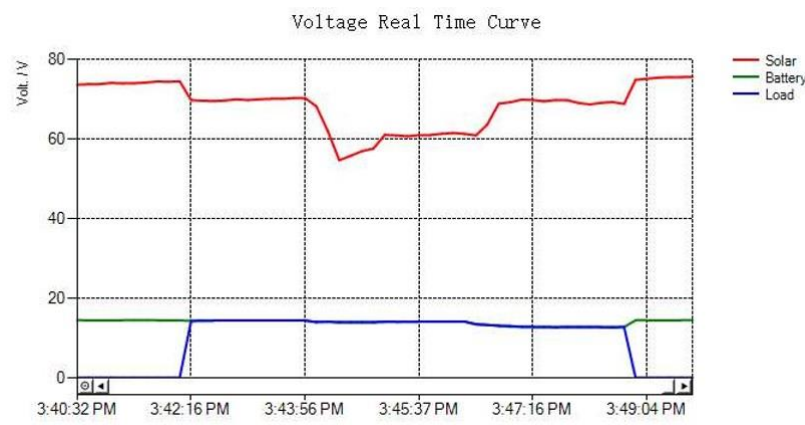

Fig. 8. Voltage real time curve. 
For the first try, because the battery is not fully charged (about 70-75\%), Boost charging mode. The charger makes the battery charge, taking power from the panel. Voltages from the battery or panel will not undergo significant changes.

Figures 9, 10 and 11 show the behavior of the controller at a battery charge level of $100 \%$.

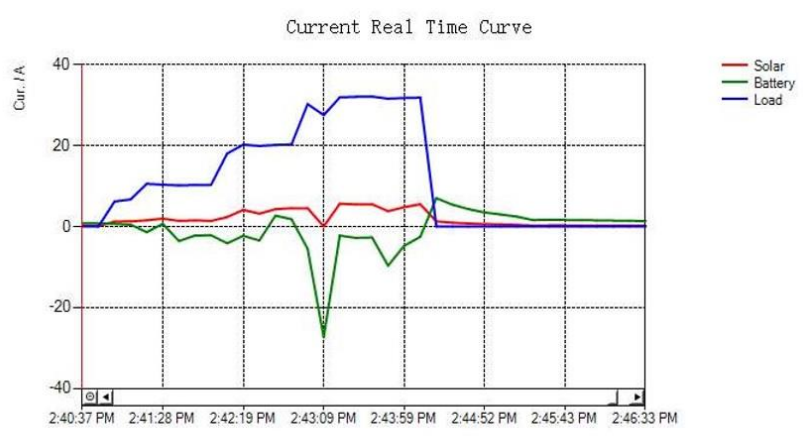

Fig. 9.Current real time curve.

Voltage Real Time Curve

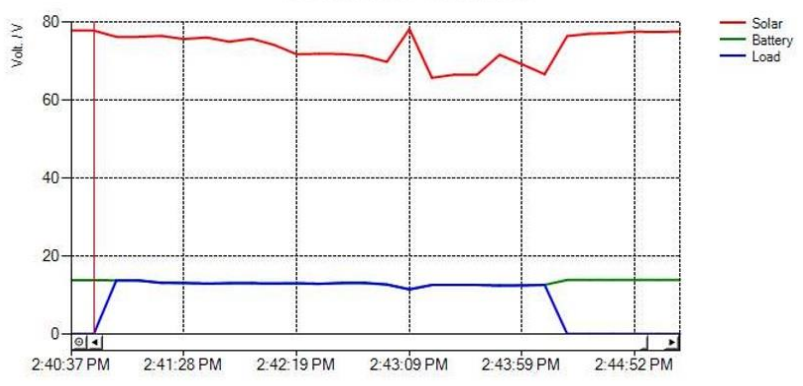

Fig. 10. Voltage real time curve.

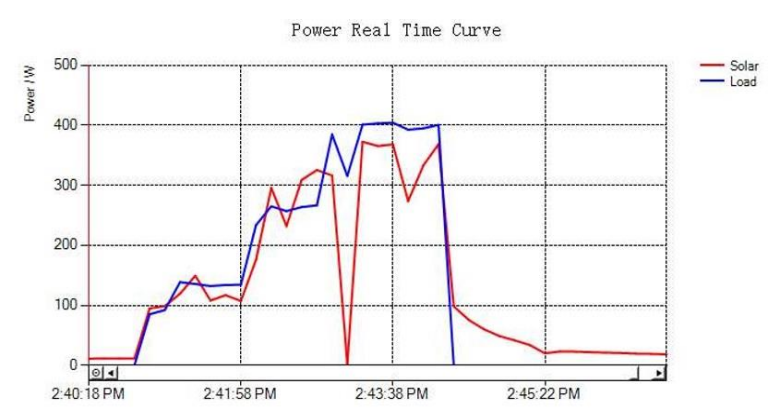

Fig. 11. Power real time curve.

At this stage, the battery is fully charged, float charging mode. This time, the panel, supplying almost all the energy required by consumers, the battery charge rate does not change.

The power graph shows a sharp drop in panel power, a phenomenon due to a cloud that reduces the amount of light reaching the photovoltaic panel, a phenomenon that takes power from the battery.

However, we notice that although the panel provides less power than consumer needs, the battery does not discharge. This does not seem possible, so we can say that it can not be seen how long the battery has been discharged due to delays in reading the parameters, with a $10 \mathrm{~s}$.

\section{CONCLUSIONS}

The automatic guidance system works as long as the light sensing light sensitivity gives a lesser signal than the signal set by a potentiometer. So with a potentiometer we can select the level of darkness until the targeting 
system works. When reaching the threshold of darkness, the system will be ordered to go home. The home position is represented by the position of the morning panel when the sunrise the sun.

Controlling the battery charge level is so important that most quality battery manufacturers (with five years or more warranty) specify the need for voltage regulation, low voltage disconnection, and temperature compensation. When these limits are not met, battery failure is expected to be four times lower than normal life expectancy, despite its quality and cost.

A solar charge controller is not costly compared to the cost of a full power system. I hope that this paper can give an idea for a wise choice in purchasing such a charger and power system.

Therefore, for those who want a safe investment over time, it is preferable that a photovoltaic power system be equipped with a solar charger to extend the life of the batteries and to get the best possible system performance.

The controller is willing to prevent complete unloading by interrupting the power supply to consumers. It can be monitored both directly with the software provided by the manufacturer, but also wirelessly using a remote controller. Given that the system works, its hysteresis is about 5 degrees, the purpose of paper was met.

\section{REFERENCES}

[1] Marinescu, D.V.N., Surse regenerabile de energie, Editura Chiminform Data, București 2004.

[2] Rajvanshi, A.K., Biomass gasification. Alternative energy in agriculture, vol. II, Ed. D. Yogi Goswami, CRC Press, 1986, p. 83-102.

[3] Deutsche Gesellschaft für sonnenenergie (Dgs), planning and installing photovoltaic systems: A guide for installers, architects and engineers (second edition), 2006, Earthscan, UK.

[4] https://en.wikipedia.org/wiki/Photovoltaics\#Photovoltaic_systems (20.03.2017).

[5] http://www.epsolarpv.com/en/ (20.03.2017).

[6] https://en.wikipedia.org/wiki/Talk:Solar_tracker\#Efficiency_increase (20.03.2017).

[7] http://www.ren21.net/Portals/0/documents/Resources/GSR/2014/GSR2014_full\%20report_low\%20res.pdf (20.03.2017). 